\title{
Masks, mechanisms and Covid-19: the limitations of randomized trials in pandemic policymaking
}

\author{
Seán M. Muller ${ }^{1}[$
}

Received: 4 January 2021 / Accepted: 14 March 2021 / Published online: 25 March 2021

(C) Springer Nature Switzerland AG 2021

\begin{abstract}
Reluctance to endorse mask wearing to slow transmission of SARSCov-2 has been rationalized by the failure of randomized control trials (RCTs) to provide supportive evidence. In contrast, a mechanism-based approach suggests that mask wearing should be expected to reduce transmission: so that contrary evidence from RCTs likely reflects the need to focus policy attention on addressing interacting or mediating factors that offset the basic positive effect. The differing conclusions that result from these two approaches reflect the limitations of RCT-based approaches that are compounded in scenarios, such as pandemics, where urgent decisions are required with limited evidence.
\end{abstract}

Keywords Covid-19 $\cdot$ Masks $\cdot$ Randomized control trials

Mask usage among the general population to mitigate the spread of SARS-Cov-2 has been one of the most contentious issues in the Covid-19 pandemic. An influential view is that mask usage should not be recommended due to ambiguous or negative findings from randomized controlled trials (RCTs). In contrast, I argue that mechanistic reasoning leads to the opposite conclusion. A simple germ theory of disease suggests that masks will have some effectiveness and therefore unsupportive

This note belongs to the Topical Collection "Seeing Clearly Through COVID-19: Current and future questions for the history and philosophy of the life sciences", edited by G. Boniolo and L. Onaga. The author is grateful to two anonymous reviewers for helpful suggestions on the manuscript.

Seán M. Muller

seanm@uj.ac.za

1 Public and Environmental Economics Research Centre (PEERC), Johannesburg Institute for Advanced Study (JIAS), School of Economics, University of Johannesburg, JBS, Empire Road, Auckland Park, Johannesburg 2092, South Africa 
findings from RCTs do not refute the case for masks per se, but instead draw attention to mediating and interacting factors that may be manipulable.

I assume throughout that reducing transmission rates is a primary objectivewhether to achieve mitigation or suppression (Ferguson et al., 2020). ${ }^{1}$ The World Health Organisation (WHO) initially declined to endorse wearing of 'medical masks' as a non-pharmaceutical intervention to prevent or reduce the spread of the SARS-Cov-2 virus and strongly opposed using other forms of masks. In the second version of the WHO's advice in March 2020 (WHO, 2020a) it stated that:

A medical mask is not required for people who are not sick as there is no evidence of its usefulness in protecting them.

Cloth (e.g. cotton or gauze) masks are not recommended under any circumstances.

However, by December 2020, the WHO recommended wearing of non-medical masks of various kind in certain community settings - subject to recommendations on how this is done. It did so, "Despite the limited evidence of protective efficacy of mask wearing in community settings" (WHO, 2020b). Various public health experts and epidemiologists have gone further, asserting that community use of non-medical masks is crucial for slowing transmission and attributing better outcomes in some countries to early adoption of widespread mask usage. Some countries that were initially reticent to even recommend mask use have made a comparable about-turn, making mask wearing mandatory and failure to do so subject to criminal penalty.

Under the currently-dominant hierarchy in evidence-based medicine, credible evidence on mask usage must come from randomized control trials; it was the absence of significant positive effects from RCTs prior to the pandemic that informed the WHO's initial stance. There are many dimensions to the disagreements about mask usage in relation to Covid-19 but here I focus on one that has been neglected in the extant literature on Covid-19: the epistemological implications of reliance on RCTs as opposed to mechanism-based reasoning. ${ }^{2}$

\section{Mechanistic reasoning versus RCTs}

The basic scientific question is: does wearing a mask in a non-medical setting reduce the probability of virus transmission, either by the wearer or to the wearer? A widelyheld view among medical scientists is that conclusive evidence requires a statistically

\footnotetext{
1 Even if masks reduce the rate of transmission they may not reduce the total number of infections over the full course of the pandemic- - that will depend on other national factors.

2 As an example of different framing, Murray (2020) proposes to explain disagreements and changes in approach by distinguishing between 'academic epidemiologists' and 'applied epidemiologists'. The former "[seek] to refine and hone a more detailed understanding of disease processes through extensive data collection, careful estimation of input parameters, and wide assessment of uncertainty", while the latter "must provide the best available advice now and update as soon as new information is available-even if that advice or information arises more from experience and intuition than scientific fact".
} 
significant finding from a high quality RCT (the 'gold standard'), but preferably from a meta-analysis of results from such RCTs. However, many concerns have been raised about the epistemic authority given to RCTs (Rothwell, 2005), particularly in the literature on evidence-based medicine, and additional concerns have been raised about the framing of meta-analysis as a 'platinum standard' (Stegenga, 2011).

For those who emphasise RCTs, without such evidence there is no basis for recommending or prescribing mask use. Mechanistic reasoning, on the other hand, does not require such agnosticism (Clarke et al., 2014). In the present case such logic may proceed as follows:

1. SARS-Cov-2 is a virus that is transmitted from one person to another

2. The main source of transmission is respiratory droplets, either in the air or on surfaces

3. Holding other factors constant, anything that reduces the volume of such droplets from contagious individuals will reduce transmission rates

4. Masks worn over the nose and mouth that are not overly porous will block transmission of some proportion of droplets

5. Therefore masks should be worn.

It is notable from the perspective of the history of medical science that this logic relies only on a fairly simple germ theory of disease. And it places the burden of proof on those who would argue against recommending face masks.

Do negative or ambiguous results from RCTs of mask usage demonstrate that this logic is incorrect? No. This is primarily because the third point contains crucial auxiliary assumptions (Lakatos, 1978). Notable among these are assumptions about behaviour. Those include two particular considerations: that the manner in which people use masks may offset the benefits; that wearing of masks may lead to less cautious behaviour in other respects. Individuals may transfer infectious material by touching their faces with unsanitized hands to place and remove a mask. In addition, or alternatively, they may be less inclined to limit social contact than if they had not been wearing a mask.

The behavioural dimension allows us to reconcile the (at best) ambiguous results from RCTs with the basic mechanism-based argument. But it does not change the policy recommendation that would typically be drawn from the RCTs: there is no basis to recommend usage of non-medical face masks in community settings. Indeed, when one Danish study appeared to find no effect of mask usage (Bundgaard et al., 2020) some researchers concluded that: "now that we have properly rigorous scientific research we can rely on, the evidence shows that wearing masks in the community does not significantly reduce the rates of infection" (Jefferson \& Heneghan, 2020). 


\section{Policy implications}

The crucial flaw in such a recommendation is that it fails to acknowledge the role of factors that affect the efficacy of masks and the corresponding possibility of altering those. As already outlined: if the basic mechanistic function of masks is accepted, then the failure of sufficiently high quality RCTs to show a significant effect is likely to be due to other factors that are offsetting this direct effect. ${ }^{3}$ But such factors may themselves be manipulable. Therefore findings from RCTs do not reflect the full spectrum of possibilities that are relevant to policy. At best, an atheoretic, RCT-dependent approach requires that more randomised interventions be conducted in which policymakers attempt to influence hypothesised mediating and interacting factors. When one considers the timelines involved it is unlikely, if not impossible, that the RCT approach can provide the necessary evidence in sufficient time. And as was noted by some researchers advocating consideration of wider mask use early in the pandemic, "there is an essential distinction between absence of evidence and evidence of absence" (Feng et al., 2020).

The mechanism-based approach presents a quite different perspective. It suggests that, for widely-accepted reasons, the default position is that masks reduce probability of transmission to some degree. If RCT evidence does not reflect this, that must be due to the influence of other factors. The appropriate policy response remains to recommend mask usage but combine such recommendations with corresponding recommendations aimed at addressing these factors. A possible counterargument is that such additional recommendations and measures are speculative, both in the factors they consider and in their prospects of efficacy. There is some truth to this, but in the specific context of the WHO's Covid-19 recommendations it is not a legitimate criticism of mask usage per se. All other non-pharmaceutical measures that were recommended — hand sanitising, social distancing and so forth—are based on a similar degree of speculative inference.

It is ironic that the absence of RCT evidence on non-pharmaceutical interventions other than masking appears to have contributed to the willingness to endorse them. Initial reluctance to endorse mask usage was based on unsupportive findings from RCTs, but that was misguided both as an epistemological matter and from a policy perspective. Mechanism-based reasoning provides a justification for the stance ultimately advocated by the WHO and adopted by many countries: wearing a mask, even of a non-medical variety, constitutes a sensible precautionary measure that may mitigate the spread of SARS-Cov-2. ${ }^{4}$

\footnotetext{
3 Where 'sufficiently high quality' here can be taken to mean that the RCT achieves internal validity (identifying the true causal effect of the intervention/treatment). In practice, RCTs may often not find true effects and this constitutes a further component of the 'protective belt' of assumptions around the basic mechanistic logic.

4 The likely extent of any mitigating effect is a more complex question that depends on characteristics of local epidemics, values of interacting and mediating factors, and time horizons.
} 


\section{References}

Bundgaard, H., Bundgaard, J. S., Raaschou-Pedersen, D. E. T., von Buchwald, C., Todsen, T., Norsk, J. B., Pries-Heje, M. M., et al. (2020). Effectiveness of adding a mask recommendation to other public health measures to prevent SARS-CoV-2 infection in danish mask wearers: a randomized controlled trial. Annals of Internal Medicine. https://doi.org/10.7326/M20-6817.

Clarke, B., Gillies, D., Illari, P., Russo, F., \& Williamson, J. (2014). Mechanisms and the evidence hierarchy. Topoi, 33(2), 339-360. https://doi.org/10.1007/s11245-013-9220-9.

Feng, S., Shen, C., Xia, N., Song, W., Fan, M., \& Cowling, B. J. (2020). Rational use of face masks in the COVID-19 pandemic. The Lancet Respiratory Medicine, 8(5), 434-436. https://doi.org/10.1016/ S2213-2600(20)30134-X.

Ferguson, N., Laydon, D., Nedjati Gilani, G., Imai, N., Ainslie, K., Baguelin, M., Bhatia, S., et al. (2020). Report 9: Impact of non-pharmaceutical interventions (NPIs) to reduce COVID19 mortality and healthcare demand. Imperial College London. https://doi.org/10.25561/77482.

Jefferson, T., Heneghan, C. (2020). Masking lack of evidence with politics. The Centre for EvidenceBased Medicine: Evidence Service to Support the COVID-19 Response (blog). 23 July 2020. https:// www.cebm.net/covid-19/masking-lack-of-evidence-with-politics/

Lakatos, I. (1978). The methodology of scientific research programmes, vol I. Philosophical Papers. Cambridge University Press

Murray, E. J. (2020). Epidemiology's time of need: COVID-19 calls for epidemic-related economics. Journal of Economic Perspectives, 34(4), 105-120. https://doi.org/10.1257/jep.34.4.105.

Rothwell, P. M. (2005). External validity of randomised controlled trials: To whom do the results of this trial apply? Lancet, 365, 82-93.

Stegenga, J. (2011). Is meta-analysis the platinum standard of evidence? Studies in History and Philosophy of Science Part C: Studies in History and Philosophy of Biological and Biomedical Sciences, 42(4), 497-507. https://doi.org/10.1016/j.shpsc.2011.07.003.

WHO. (2020a). Advice on the use of masks in the community, during home care, and in health care settings in the context of COVID-19. WHO/2019-nCoV/IPC_Masks/2020.2. World Health Organisation

WHO. (2020b). Mask use in the context of COVID-19. WHO/2019-nCoV/IPC_Masks/2020.5. World Health Organisation

Publisher's Note Springer Nature remains neutral with regard to jurisdictional claims in published maps and institutional affiliations. 